\title{
Delayed Hypoxia During Pediatric Posterior Spinal Fusion Secondary to Intraoperative Pneumothorax: Case Report
}

\author{
Michelle Rovner ${ }^{1}$, Zachary Jeanes ${ }^{1}$, Amanda Redding ${ }^{1}$, Grayce Davis $^{1}$, and Cory Furse ${ }^{1}$ \\ ${ }^{1}$ Medical University of South Carolina
}

May 6, 2020

\begin{abstract}
Posterior spinal surgery has the risk of perioperative respiratory complications. We describe the delayed diagnosis of an intraoperative pneumothorax in a teenage girl with idiopathic scoliosis scheduled for thoracolumbar posterior fusion. After an uneventful surgery, the oxygen saturation abruptly decreased to $65 \%$. A chest x-ray showed a moderate pneumothorax.
\end{abstract}

\section{Key Clinical Message}

Delayed severe hypoxia secondary to an intraoperative pneumothorax without any intraoperative respiratory complications is an extremely rare event. These unusual clinical signs must be recognized and treated to maintain the patient's safety.

\section{Introduction}

Intraoperative pneumothorax is a rare and potentially catastrophic clinical phenomenon that can be quite challenging to diagnose during a surgical procedure. A delay in presentation makes the situation even more complicated and unusual. In the general pediatric population, the overall incidence of spontaneous pneumothorax is 3.4/100,000 patients, with males having a 10-fold greater incidence than females. ${ }^{1,2}$ The incidence of pneumothorax in the perioperative period can be variable based on the type of surgical procedure.

Posterior spinal surgery is associated with the risk of several perioperative respiratory complications including pleural effusion, pneumothorax, hemothorax, pulmonary edema, chylothorax, and prolonged mechanical ventilation. ${ }^{3}$ During deep dissection between the transverse processes or instrumentation and placement of pedicle hardware, an inadvertent tear in the parietal pleura can result in entrainment of outside air into the pleural space. Differentiating between a pneumothorax and the myriad of other potential intraoperative respiratory or airway issues is difficult, and can delay accurate diagnosis. Treatment of a pneumothorax in the prone position is complicated by limited access to the typical sites of pleural decompression. In this report, we describe an unusual presentation of a pneumothorax in a pediatric patient with idiopathic scoliosis undergoing posterior spinal fusion. Written Health Insurance Portability and Accountability Act authorization was obtained from both the patient and parent.

\section{Case Report}

A 15 year-old, $68 \mathrm{~kg}$ female with a history of idiopathic scoliosis was scheduled for thoracolumbar posterior fusion and instrumentation (levels T3-L4). Additional past medical and surgical history were unremarkable, without allergies or chronic medications. Induction of general anesthesia was achieved with intravenous lidocaine, Propofol, fentanyl, and succinylcholine. Following easy mask ventilation, the patient was intubated uneventfully via direct laryngoscopy with a 7.0 cuffed endotracheal tube. Total intravenous anesthesia (TIVA) with Propofol, remifentanil, and dexmedetomidine infusions was utilized for maintenance. Additionally, infusions of aminocaproic acid and nicardipine were employed for fibrinolysis and intermittent 
hypertension, respectively. The patient was turned to the prone position with no change in oxygenation or ventilation and all other vital signs were stable.

Ventilator settings included fraction of inspired oxygen (FiO2) 0.4, tidal volume (TV) 470cc, respiratory rate (RR) 11, and positive end expiratory pressure (PEEP) $5 \mathrm{cmH}_{2} \mathrm{O}$. Four hours post-induction, the first arterial blood gas (ABG) analysis showed 7.42/33/231/22/-3/99\% with hemoglobin (Hgb)/hematocrit (Hct) $=8.5 / 25$. Estimated blood loss (EBL) at that time was approximately 500cc. One hour later, the surgeon noticed cerebrospinal fluid in the operative field resulting from a dural tear at T2-T3. A pediatric neurosurgeon was consulted and a lumbar drain was placed at L2-L3. During this period, the patient became mildly hypotensive with a blood pressure (BP) of $93 / 53 \mathrm{mmHg}$, however oxygenation and ventilation remained stable. At this point, 1000cc of 5\% albumin and 2500cc of crystalloid had been administered along with a transfusion of 60cc Cell Saver. Later on a second ABG analysis was obtained with normal results except for a low hematocrit: 7.43/37/218/24/0/99\% with Hgb/Hct $=6.1 / 18$. Two units packed red blood cells were transfused, the blood pressure stabilized, and the surgeon began closing. While still prone, the patient was transitioned to pressure support (PS) ventilation with settings of PS 14, PEEP 5, FiO2 0.4, RR 17-20, achieving tidal volumes of $350 \mathrm{cc}$. The FiO2 was then increased to 1.0 in preparation for returning to supine position and emergence. All infusions were discontinued prior to turning supine, and acetaminophen $1000 \mathrm{mg}$, hydromorphone $0.6 \mathrm{mg}$, fentanyl $200 \mathrm{mcg}$, were administered intravenously. Total fluids given for the case were 1000cc albumin 5\% and 4000cc crystalloid, 60cc Cell Saver and 2 units packed red blood cells. Urine output was $3475 \mathrm{cc}$ with an EBL of $1000 \mathrm{cc}$.

By the end of the case, the patient had been spontaneously ventilating with pressure support during wound closure for 30 minutes. The respiratory rate was 17-20 breaths/minute and TV 350-450cc. All vital signs were normal. Residual neuromuscular blockade was reversed, and the patient was turned supine. Immediately upon turning supine, her pulse oxymetry decreased to $65 \%$ with mild hypotension (BP 96/40) and a heart rate (HR) of 80. She became tachypneic (RR 30) with TV decreased to around 150cc. Respirations were supported with intermittent manual ventilation at an $\mathrm{FiO} 2$ of 1.0 over the next three minutes. This resulted in a slow, but steady increase in oxygenation to $89 \%$. Over the next 5 minutes the oxygenation continued to rise slowly to $96 \%$. Breath sounds were equal bilaterally without wheezing at the apices anteriorly, but were difficult to auscultate posteriorly. After 30 minutes, the patient was awake and appropriately following commands with unsupported TV of 250-275cc, RR 30, BP 121/53, HR 92, and oxygen saturation of 97\%. The patient was extubated, after which, she immediately desaturated to $83 \%$ but was otherwise hemodynamically stable. Ventilation was assisted via bag-mask, but she remained tachypneic achieving poor TV (approximately $200 \mathrm{cc}$ ) with good mask seal. A stat portable chest x-ray revealed a moderate right-sided pneumothorax. ABG analysis showed hypoxemia and respiratory acidosis $7.28 / 53 / 89 / 25 /-2 / 96$, Hgb/Hct=8.2/24. The patient was sedated in the operating room and the surgical team, under local anesthesia, placed a chest tube. The patient's oxygenation immediately improved to $100 \%$. She was then transported to the intensive care unit. The chest tube was removed on post-op day (POD)\#1, her lumbar drain was removed POD\#2, and the remainder of her hospital course proceeded uneventfully with discharge on POD\#5. The patient and family were very satisfied with their care.

\section{Discussion}

Intraoperative pneumothorax is a rare, albeit known, complication of posterior spinal surgery possibly that can result from a difficult dissection, aberrant probe or screw placement, or barotrauma. Initial management typically consists of evacuating the pneumothorax by needle aspiration, percutaneous placement of a drainage catheter, or tube thoracostomy. Prone positioning during scoliosis surgical procedures may affect access to those intervention sites.

Although certainly on the differential for any intraoperative respiratory problem, the development of a pneumothorax is primarily a clinical diagnosis that relies on a high index of suspicion. Some clinical findings suggestive of a pneumothorax include sudden increased airway pressures, unexplained hypoxia, rising end tidal carbon dioxide, and unilateral absence of breath sounds to auscultation. Under positive pressure mechanical ventilation, intrapleural pressures can rapidly increase from an unrecognized pleural defect to 
become a tension pneumothorax. This can be life threatening, resulting in quick deterioration including tachycardia, tachypnea, hypotension, and tracheal and cardiac deviation leading to severe hemodynamic compromise and cardiopulmonary collapse. ${ }^{4}$

Interestingly, we observed none of these clinical signs throughout the procedure until the end of the case when turning from prone back to supine position. There were no airway or pulmonary issues that occurred prior to that incident that would suggest a pneumothorax. One possible explanation is that a pleural tear occurred during hardware placement and the increased intrathoracic pressure was relieved during the open/prone portion of the case. Thus air trapping only manifested once the wound was closed and the patient returned to supine position. Additionally, the development of the pneumothorax may have progressed slowly due to the patient spontaneously breathing during wound closure with pressure support ventilation. Outside of known pleural tears, there have also been reports of spontaneous pneumothorax (without evidence of intraoperative pleural injury) in the perioperative period following posterior spinal fusion surgery, with a reported incidence 0.2 to $1.6 \% .^{5-7}$ Potential risk factors for the development of an intraoperative pneumothorax include blebs or bullae, or longer amounts of time in the chest with multiple passes and probing. In the absence of a known pleural injury or these risk factors, potential causes of a pneumothorax in the perioperative period are few. Re-expansion of an occult pneumothorax or damage from elevated peak inspiratory pressures are possibilities. Of note, underlying pulmonary dysfunction has not been associated with an increase in the overall risk of pulmonary complications during spinal deformity surgery. ${ }^{8}$

Our differential diagnosis for the hypoxia consisted of atelectasis, inadequate minute ventilation, pain, endotracheal tube migration, fluid overload, mucous plug, neuromuscular weakness, and pneumothorax. Although pneumothorax is not usually high on the differential for hypoxia in most circumstances in the operating room, the hypoxia that occurred in our patient was acute and severe. Fortunately, the timing of our patient's respiratory distress opened several diagnostic and therapeutic options that would have been difficult in the prone position. We ruled out other potential causes and then quickly obtained a portable chest radiograph confirming a pneumothorax. Transthoracic ultrasound is another valuable tool used to pare down the list of diagnoses in a hypoxic patient, potentially leading to a timelier intervention. ${ }^{9}$ Unfortunately, we are unable to discern the etiology of the pneumothorax in our patient but it most likely resulted from a difficult dissection. Our surgical team never had any indication that a pleural injury occurred.

The delayed hypoxia in this patient is an unusual event related to an intraoperative pneumothorax. Having had no indication of any respiratory problems throughout the case and then to have severe and acute hypoxia on emergence is a rare complication. It is therefore imperative to recognize the clinical signs that may warrant an intervention to maintain the patient's safety.

\section{References}

1. Lopez ME, Fallon SC, Lee TC, Rodriguez JR, Brandt ML, Mazziotti MV. Management of the pediatric spontaneous pneumothorax: is primary surgery the treatment of choice? The American Journal of Surgery . 2014;208(4):571-576.

2. Dotson K, Johnson LH. Pediatric spontaneous pneumothorax. Pediatric Emergency Care . 2012;28(7):715-720.

3. Rajmani V, Bhagawati D, Noordeen H, Lehovsky J, Gibson A. Incidence of early pulmonary complications requiring chest tube insertion in surgically treated paediatric scoliosis. The Spine Journal . 2017;17(3):5.

4. Leigh-Smith S, Harris T. Tension pneumothorax-time for a re-think? Emergency Medicine Journal: emj . 2005;22(1):8-16.

5. Yin S, Tao H, Du H, et al. Postoperative pulmonary complications following posterior spinal instrumentation and fusion for congenital scoliosis. Plos One . 2018;13(11):0207657.

6. Li G, Lv G, Passias P, et al. Complications associated with thoracic pedicle screws in spinal deformity. European Spine Journal . 2010;19(9):1576-1584.

7. Suk SI, Kim WJ, Lee SM, Kim JH, Chung ER. Thoracic pedicle screw fixation in spinal deformities: are they really safe? Spine . 2001;26(18):2049-2057. 
8. Liang J, Qiu G, Shen J, et al. Predictive factors of postoperative pulmonary complications in scoliotic patients with moderate or severe pulmonary dysfunction. Journal of Spinal Disorders 83 Techniques . 2010;23(6):388-392.

9. Ueda K, Ahmed W, Ross AF. Intraoperative pneumothorax identified with transthoracic ultrasound. Anesthesiology . 2011;115(3):653-655. 